\title{
CONCEPÇÃO SOBRE A INVESTIGAÇÃO NO ENSINO DE CIÊNCIAS
}

\author{
DISEÑO DE LA INVESTIGACIÓN EN LA ENSEÑANZA DE LAS CIENCIAS
}

RESEARCH DESIGN IN SCIENCE EDUCATION

João Paulo Cunha de MENEZES ${ }^{1}$

RESUMO: A literatura científica tem enfatizado a importância das experiências de investigação para os estudantes na formação inicial de professores. Tentando melhor compreender este cenário, o objetivo deste trabalho foi investigar a percepção dos estudantes de Licenciatura em Ciências Biológicas em uma atividade prática no Ensino de Ciências baseado em investigação. Para alcançar este objetivo os estudantes foram acompanhados em duas disciplinas práticas durante cinco semestre. Durante o desenvolvimento das disciplinas os estudantes foram estimulados a manterem anotações sobre todo o processo. Ao final da disciplina foi solicitado um relato das atividades desenvolvidas. Estas informações foram utilizadas como material de análise neste trabalho. Os dados obtidos sugerem que as concepções dos estudantes sobre o processo de pesquisa e investigação na educação estavam relacionadas à prática e interpretação de seu próprio projeto de investigação e que a vivência do projeto modificou as concepções que os mesmos possuíam sobre a pesquisa. Este trabalho apresenta que as experiências vivenciadas na formação inicial docente instigam uma maior reflexão sobre a natureza da investigação. Desta forma, acredito que os resultados aqui apresentados podem contribuir na formação inicial destes estudantes.

PALAVRAS-CHAVE: Formação inicial de professores. Práticas pedagógicas. Ensino por investigação.

RESUMEN: La literatura cientifica ha destacado la importancia de las experiencias de investigación para los estudiantes en la formación inicial del profesorado. Tratando de comprender mejor este escenario, el objetivo de este trabajo fue investigar la percepción de los estudiantes de grado en una actividad práctica en la enseñanza de las ciencias basada en la investigación. Para lograr este objetivo se acompañó a los estudiantes en dos disciplinas prácticas durante cinco semestres. Durante el desarrollo de las disciplinas se animó a los estudiantes a tomar notas sobre todo el proceso. Al final de la disciplina se solicitó un informe de las actividades desarrolladas. Esta información se utilizó como material de análisis en este trabajo. Los datos obtenidos sugieren que las concepciones de los estudiantes sobre el proceso de investigación e indagación en educación estaban relacionadas con la práctica e interpretación de su propio proyecto de investigación y que la experiencia del proyecto modificó sus concepciones sobre la investigación. Este trabajo presenta que las experiencias vividas en la formación docente inicial instigan una mayor reflexión sobre la

${ }^{1}$ Universidade de Brasília (UnB), Brasília - DF - Brasil. Professor Adjunto, Núcleo de Educação Científica, Instituto de Ciências Biológicas. Doutorado em Recursos Hídricos (UFLA). ORCID: https://orcid.org/00000002-2566-3957. E-mail: jpaulo_bio@hotmail.com

RIAEE - Revista Ibero-Americana de Estudos em Educação, Araraquara, v. 16, n. 4, p. 2872-2887, out./dez. 2021. e-ISSN: 1982-5587 
naturaleza de la investigación. De este modo, creo que los resultados presentados aquí pueden contribuir a la formación inicial de estos alumnos.

PALABRAS CLAVE: Formación inicial del profesorado. Prácticas pedagógicas. Enseñar por la investigación.

ABSTRACT: The scientific literature has emphasized the importance of research experiences for students in initial teacher formation. Trying to better understand this scenario, the objective of this work was to investigate the perception of teaching degree students in Biological Sciences in a practical activity in research-based Science Teaching. To achieve this objective the students were accompanied in two practical disciplines during five semesters. During the development of the discipline's students were encouraged to keep notes about the whole process. At the end of the course an account of the activities developed was requested. This information was used as analysis material in this work. The data obtained suggest that the students' conceptions about the research and investigation process in education were related to the practice and interpretation of their own research project and that the experience of the project modified their conceptions about the research. This work presents that the experiences lived in the initial teacher formation instigate a greater reflection on the nature of research. In this way, I believe that the results presented here can contribute to the initial formation of these students.

KEYWORDS: Initial teacher training. Pedagogical practices. Teaching by research.

\section{Introdução}

As atuais reformas no Ensino de Ciências no Brasil exigem cada vez mais mudanças na forma como o estudante aprende. Para a maioria dos professores, isso requer refletir sua própria prática e desenvolver novos papéis tanto para eles, como professor, quanto para os estudantes (DARLING-HAMMOND; MCLAUGHLIN, 1995). Nesta perspectiva, Predebon e Pino (2016) destacam a importância de inserir na formação inicial de professores práticas que possuam um caráter investigativo. De acordo com essa abordagem, a investigação pode servir como um meio de envolver os estudantes nas ideias, conceitos, processos e práticas da ciência (ADLER et al., 2018). Embora não seja a única maneira de Ensinar Ciências, acredita-se que o ensino baseado em investigação tenha uma poderosa influência no aprendizado dos estudantes (NRC, 2000).

O ensino baseado em investigação abrange um amplo espectro de abordagens, que vão desde a pesquisa estruturada e guiada dirigida pelo professor até a pesquisa aberta dirigida ao estudante (NRC, 2000; MUNFORD; LIMA, 2007; SASSERON, 2018). Na investigação estruturada, os estudantes investigam uma pergunta apresentada pelo professor por meio de 
um procedimento prescrito, levando a um resultado predeterminado, semelhante a seguir uma receita (KABERMAN; DORI, 2009). Diferentemente da pesquisa estruturada, na pesquisa guiada os estudantes investigam questões e procedimentos que os professores apresentam a eles, mas os próprios estudantes decidem sobre os processos a serem seguidos e podem chegar a conclusões imprevisíveis, mas bem concebidas (BLANCHARD et al., 2010).

A aprendizagem baseada em investigação pode facilitar a aquisição de conhecimento e compreensão conceitual e processual como a promoção de habilidades de pensamento flexíveis e práticas epistêmicas (HMELO-SILVER; DUNCAN; CHINN, 2007; MUNFORD; LIMA, 2007; SASSERON, 2018). No entanto, a aprendizagem baseada em investigação também apresenta numerosos desafios para os estudantes, tendo os professores com papel fundamental na facilitação deste processo (HMELO-SILVER; DUNCAN; CHINN, 2007). Crawford (2000) expandiu esse escopo e afirmou que o professor em uma sala de aula baseada investigação deve assumir uma infinidade de funções, como motivador, diagnosticador, guia, inovador, experimentador, pesquisador, modelador, mentor e colaborador.

Apesar do papel central da investigação no processo de ensino-aprendizagem, o ensino baseado neste processo raramente é implementado em sala de aula (WINDSCHITL, 2003; MUNFORD; LIMA, 2007; CAPPS; CRAWFORD; CONSTAS, 2012). Desta forma muitos professores acabam usando o modelo tradicional de ensino, refletindo a forma como foram ensinados, no entanto pode haver outras razões para ausência destas atividades, tais como: crenças tradicionais sobre o processo ensino-aprendizagem (SAAD; BOUJAOUDE, 2012), falta de habilidades pedagógicas (SHULMAN, 2013), falta de tempo (LOUGHRAN, 1994), conhecimento inadequado da prática (SAAD; BOUJAOUDE, 2012) e formação inadequada. À luz dessas descobertas, uma das tarefas mais difíceis que os cursos de Licenciatura enfrentam é desenvolver a capacidade dos docentes em apoiar seus estudantes durante estas atividades. Na intenção de superar essas dificuldades de formação docente Harres et al. (2005, p. 2) afirma que:

[...] pesquisadores na área, oriundo de diferentes contextos, e até as novas diretrizes para a formação de professores no Brasil indicam a necessidade de implementação de uma perspectiva investigativa na formação inicial. Porém, essas inovações não estão prontas em lugar nenhum.

O aprendizado relacionado a atividades investigativas que os estudantes de Licenciatura possuem vem das experiências dos cursos de graduação. Assim como acontece na educação pré-escolar, o docente de nível superior não apenas ensina o conteúdo de seus 
cursos, mas também modela práticas e estratégias de ensino nos futuros professores em suas aulas (GROSSMAN; WILSON; SHULMAN, 1989). Desta forma, os estudantes precisam de instruções mais explícitas sobre o Ensino de Ciência por investigação, incluindo como criar conhecimento por meio de argumentos baseados em explorações e evidências (ROGERS, 2009). Desta forma, a construção destas competências nos professores é essencial para aumentar a alfabetização científica nos estudantes, consistindo em uma compreensão significativa de conceitos e habilidades científicas (MUNFORD; LIMA, 2007; BHATTACHARYYA; VOLK; LUMPE, 2009; SASSERON, 2018), como também no interesse pela ciência (LEE et al., 2008). Para evitar o ciclo contínuo de retrato negativo ou impreciso da ciência nas salas de aula do Ensino Fundamental e Médio, é imperativo que os programas de formação de professores preparem estudantes de maneira mais eficaz, incorporando experiências autênticas de investigação nos cursos de Licenciaturas, para que não sejam introduzidos superficialmente na investigação (BAXTER et al., 2004)

Desta forma, disciplinas que forneçam experiências de investigação na formação pedagógicas dos licenciandos são de extrema importância na formação docente para que os mesmos possam construir sua própria identidade profissional e desenvolver tais atividades em sala de aula (BREDA et al., 2016; CUNHA et al., 2016; STAFFORD, 2016; DATTEIN; GÜLLICH; ZANON, 2018). Professores de disciplinas de práticas de ensino, por exemplo, podem oferecer oportunidades para que os estudantes em formação conduzam suas próprias atividades investigativas no ensino e vinculem suas experiências em sala de aula (exemplo; OLIVEIRA; SCHNEIDER, 2016). Tais disciplinas se configuram num locus importante de pesquisa sobre as práticas pedagógicas dos licenciandos. Aliar as ações propostas pelas disciplinas citadas às práticas investigativas parece ser um importante caminho.

Com base nos tópicos abordados, o objetivo deste trabalho foi investigar a percepção dos estudantes de Licenciatura em Ciências Biológicas em uma atividade prática no Ensino de Ciências baseado em investigação. Este trabalho também analisou como essas experiências podem contribuir com a prática em sala de aula. Para tanto, pretendo responder as seguintes perguntas: i) Qual o ponto de vista dos estudantes de Licenciaturas referente à investigação na sala de aula?; ii) Qual a contribuição das experiências de investigação na mudança de concepções dos estudantes de licenciatura? iii) Disciplinas de Práticas de Ensino ajudam os estudantes de licenciatura a adquirir habilidades no Ensino de Ciências baseado em investigação? 


\section{Metodologia}

Este trabalho caracteriza com uma abordagem de estudo de casos múltiplos a fim de dar sentido às relações entre as concepções e ações dos sujeitos em relação à investigação de suas ações (HUBERMAN; MILES, 1994). Os participantes da pesquisa para o desenvolvimento das atividades correspondem a estudantes matriculados nas disciplinas de Práticas de Educação em Biologia 1 e Práticas de Educação em Biologia 2 dispostos ao longo dos anos de 2017 a 2019 (cinco semestres, 8 turmas) do curso de Licenciatura em Ciências Biológicas da Universidade de Brasília. As disciplinas de Práticas de Ensino em Biologia 1 e 2 constitui disciplinas com atividades práticas desenvolvidas em grupos nos quais os estudantes propõem e elaboram um projeto de pesquisa de educação a ser implementado em sala de aula com caráter investigativo, que possa ser concluído em até seis meses. Os estudantes no início da disciplina são informados que o trabalho culmina em uma apresentação final para toda a turma e que tem por objetivo defender a proposta elaborada (tema, problema, metodologia, resultado e conclusões).

Como critério de avaliação e análise das disciplinas: i) os estudantes foram estimulados a manter anotações coletadas ao longo de seus projetos, enfatizando todos os elementos que consideraram importante nesta vivência; ii) durante a última semana da disciplina, os estudantes foram convidados a enviar um relato escrito no qual descrevem de forma individual e livre o que consistiu trabalhar a investigação em sala de aula e qual a contribuição que estas práticas de ensino acarretaram em sua formação; e iii) foi solicitado aos estudantes no final da disciplina uma reflexão apresentando os pontos positivos e negativos relacionados com o projeto, possíveis implicações para sua formação docente e suas percepções sobre sua própria experiência de investigação e o processo de implementação de uma intervenção investigativa. Todos os relatos foram enviados por meio da plataforma digital Google forms, de forma que os estudantes tiveram total liberdade para opinar (42 relatos enviados). Todo material apresentado foi utilizado para responder aos objetivos propostos.

A análise dos dados foi efetuada com base nos pressupostos teóricos de Bardin (2011) para análise de conteúdo. Nota-se que, de forma resumida, nesse tipo de análise, inicialmente todo o material produzido é lido e após, procura-se por palavras, temas, expressões que possam configurar-se em categorias de análise, que posteriormente são discutidas e interpretadas em confronto com a literatura (BARDIN, 2011). As categorias da pesquisa emergiram dos dados contidos nos relatos, partindo das reflexões dos estudantes sobre a visão 
da pesquisa no Ensino de Ciências e suas relações com as disciplinas de práticas. A análise resultou em 3 categorias: i) a primeira seção descreve as reflexões dos Licenciandos e suas respetivas interpretações da experiência acumulada durante o trabalho de investigação no Ensino de Ciências, ii) a segunda apresenta as compreensões da investigação no Ensino de Ciências, iii) e por fim, a terceira relata as associações das disciplinas práticas com o exercício de uma atividade de investigação. De forma a preservar o anonimato dos estudantes, os relatos foram denominados pela letra "EST" (referente a estudante) seguida pela numeração.

\section{Resultados e Discussões}

Em relação às vivências ao longo do processo na formação docente, a maioria dos licenciandos $(37,82 \%)$ relatou ter três tipos de experiências relacionadas ao uso da investigação em sala de aula. No entanto, os demais licenciandos $(5,18 \%)$ apresentaram opiniões e conceitos errôneos sobre esta metodologia. Esses estudantes apresentaram reflexões relacionando o processo de investigação a outros métodos de ensino (tais como atividades práticas, experimentais), sugerindo que exista confusão com o termo. Essas ideias se relacionam com muitos dos conceitos errados e mitos que os educadores têm sobre a investigação (NRC, 2000).

As experiências citadas quanto às vivências foram: i) dificuldades iniciais na proposição de uma investigação; ii) superação das dificuldades; e iii) vivenciar uma sensação de execução da atividade. No entanto, os relatórios apresentados variaram em termos da intensidade com que os sentimentos foram descritos.

$\mathrm{Na}$ fase inicial da implementação do projeto os licenciandos descreveram experiências predominantemente pessimistas associadas ao seu sentimento de dificuldade na implementação da atividade, devido à inexistência entre uma prática real aplicada e o conhecimento teórico. Essas experiências podem preencher a lacuna entre a teoria pedagógica (conteúdo) e a prática de ensino (ADLER; ZION; RIMERMAN-SHMUELI, 2019). Os licenciandos também se depararam com a falta de preparo prático para administrar uma sala de aula, conforme pode ser observado:

[...] quando começamos a trabalhar a temática na sala de aulas, os alunos estavam muitos ansiosos o que gerou muita conversa e discussão durante o desenvolvimento da atividade... (EST 14)

Houve muita discussão sobre formação de grupo, troca de colegas, escolha de lugares [...] a falta de disciplina acabou acarretando muito tempo perdido em sala de aula para que se conseguisse chegar em um consenso... (EST 23) 
[...] a falta de disciplina dos alunos atrapalhou o rendimento das aulas. A organização do espaço acabou ocupando mais tempo do que a proposta de atividade e, portanto, tivemos prejuízos na implementação no primeiro dia... (EST 27)

A partir da análise das reflexões individuais, foi possível verificar que este sentimento está intimamente ligado à etapa de formulação de hipóteses, na qual, além disso, se observa uma menor percepção de aprendizagem. Nestes momentos da disciplina, parece lógico encontrar uma certa "insegurança", que pode estar relacionada com a falta de confiança nas suas capacidades docentes. Por este motivo, é necessário abordar explicitamente nos programas de formação docente, como foi feito no presente estudo, as atividades de investigação. Quanto à percepção exercida pelos licenciandos nessa etapa inicial, suas reflexões indicaram falta de preparo para gerir a sala de aula.

Sinto que passei pela atividade de maneira bastante direta, mas fiz muitos ajustes no começo da atividade e no final acabei fazendo mais perguntas. No começo da atividade acabei por optar por poucas perguntas, ... questionando e observando os alunos, mas não funcionou... (EST 4)

Eu pensei que trabalhar uma pergunta com os estudantes seria dificil, mas conseguir um retorno por parte deles ainda foi pior... (EST 27)

Eu pensei muito em como exatamente eu descreveria a construção de hipótese com os alunos... pensei com as experiências que tive na universidade as possibilidades enfatizam a necessidade de uma resposta que não fosse respondida com "sim ou não" (EST 31)

O licenciando EST 27 também sugeriu que, embora os estudantes de ensino médio tenham dificuldade em criar hipóteses, deve-se aproveitar os questionamentos gerado por eles e tentar levar ao pensamento de uma "hipótese", sem caracterizá-la, enfatizando a importância de trabalhar a ciência com base na investigação, "você não pode simplesmente saber se é uma boa hipótese... espera-se que seja testada" (EST 27). Outro ponto elencado neste momento foi que tais atividades exigiam um planejamento inicial mais intenso, diferente do modo como planejavam aulas tradicionais no estágio "[...] trabalhar desta forma é muito mais complicado que trabalhar com aulas tradicionais..." (EST 31).

Apesar de inúmeras publicações que sustentam as vantagens de uma abordagem investigativa (CHEN et al., 2014; MARSHALL et al., 2016), sua prática de ensino ainda continua sendo um desafio. A falta de experiências que permitam uma implementação em sala de aula ainda é marcante, considerando a ênfase que o ensino de investigação tem recebido nas reformas educacionais nas últimas décadas (NRC, 2000). Além disso, nos poucos casos em que essa abordagem é aplicada, o equívoco do ensino por investigação e da natureza da ciência culmina em uma implementação deficiente (MCLAUGHLIN; MACFADDEN, 2014). 
Outros fatores identificados como dificuldades na utilização dessa abordagem também são destacados, como os processos de planejamento e avaliação, a gestão da sala de aula ou os recursos e restrições do sistema educacional (ROMERO-ARIZA et al., 2020), conforme foi retratado acima.

Após a etapa inicial do projeto, os licenciandos relataram um maior tempo gasto para poder lidar com as dificuldades que estavam enfrentando em relação à aplicação de tuas atividades em sala de aula. À medida que o projeto se desenvolvia os licenciandos compreendiam a necessidade de uma maior organização e planejamento para executar a atividade. Os licenciandos com a ajuda do professor regente intervieram na falta de disciplina dos estudantes, mediaram questões de conflitos que surgiram no decorrer da aplicação e se apropriaram da ideia do papel fundamental que exerciam naquele momento.

Observei que, apesar de eu ser um aluno de licenciatura os estudantes do ensino básico nos veem como regentes da atividade, cabendo a mim a organização da turma...os alunos possuem uma idade baixa o que implica a necessidade de uma pessoa para coordenar suas ações... (EST 4)

Nesse momento, as reflexões negativas dos licenciandos foram gradualmente reduzidas e substituídas por experiências positivas. Em suma, a experiência de superar dificuldades pode ser vista como um ponto de guinada na percepção dos licenciandos. Assim, podemos inferir que eles começam a compreender que o sucesso da aplicação era basicamente de sua responsabilidade, sendo alcançado com esforços, conhecimentos e habilidades adquiridas. Nesse estágio, suas dúvidas foram substituídas por uma maior confiança.

No meio do desenvolvimento das atividades, a maioria dos licenciandos perceberam uma maior desenvoltura nas atividades, compreendendo algumas vantagens de trabalhar a investigação no Ensino de Ciências. Neste momento, os licenciandos passaram a se sentir confiantes pela aplicação. Um dos aspectos observados nesta fase de desenvolvimento da atividade foi a oportunidade de estabelecer relações pessoais com os estudantes.

Trabalhar com atividades investigativas no ensino médio foi bastante interessante e agradável, por meio das discussões geradas para criar e responder as hipóteses levantadas foi possivel eu ajudar os alunos de forma individual. Eu era capaz de conduzir o trabalho do pensamento científico da mesma forma como meu orientador faz comigo... (EST 14)

Esses resultados parecem coesos, se for considerado que talvez os participantes não tenham tido o momento/oportunidade de realizar atividades de pesquisa durante seus estágios obrigatórios, tendo em vista que esta abordagem ainda não está difundida na prática educacional (VÁZQUEZ; MANASSERO, 2015). Diferente do que foi observado por Adler; 
Zion e Rimerman-Shmueli (2019), os resultados demostram que as reflexões dos licenciandos no entendimento processual, na aprendizagem como processo e no ponto de vista afetivo foram de moderadas para altas. Essas observações sugerem a capacidade dos licenciandos em apoiar efetivamente esses aspectos enquanto orientam a atividade.

$\mathrm{Na}$ fase final do trabalho, foi observado que quase todos os licenciandos possuíam um sentimento de "dever cumprido" após as mudanças que ocorreram em suas aulas. A disciplina dos estudantes, que anteriormente foi colocada como obstáculo para a execução da atividade, foi alterada. Segundo as reflexões apresentadas, com o desenvolvimento da atividade os licenciandos conseguiram gerir a turma de forma mais eficaz, conduzindo assim o trabalho. Desta forma é possível deduzir que os licenciandos se tornaram mais conscientes do processo da investigação científica, contribuindo também para uma melhor gestão da sala de aula.

Uma outra categoria emergente foi a "compreensão da investigação no Ensino de Ciências". Nesta categoria foi possível inferir que ouve uma maior compreensão da atividade durante a vivência. Os estudantes de licenciatura refletiram sobre o impacto das atividades investigativas em seus próprios conhecimentos e atitudes com relação ao Ensino de Ciências. Embora tenham simulado diferentes atividades em sala de aula, essa compreensão ocorreu principalmente ao final das atividades, momento no qual começaram a desenvolver uma compreensão maior do processo, conforme pode ser observado pelo seguinte relato:

\begin{abstract}
Desde o inicio da atividade tive autonomia do meu projeto. A capacidade de escolher um tema no qual me interessava para trabalhar com os alunos me motivou a me comprometer com minhas observações, mas foi apenas no final que pude compreender melhor tudo aquilo que estava desenvolvendo (EST 22)

[...] diferente das experiências de formato de aulas que tive desde o ensino fundamental até o ensino superior, no qual haviamos somente aulas expositivas, uma aula com investigação foi um desafio. Desafio pois nunca havia tido nada semelhante, mas me revelou um método eficaz de tratar temas que nem sempre são agradáveis para os alunos..., foi ao final do meu projeto que consegui a liderança da turma e pude compreender as habilidades que são desenvolvidas neste tipo de prática (EST 40)
\end{abstract}

Essas reflexões estão alinhas a outros autores (KAZEMPOUR, 2013), indicando que a imersão dos acadêmicos em experiências de sala de aula pode ser uma forma de aumentar seus conhecimentos e habilidades na investigação científica, bem como sua confiança e motivação para tratar de uma atividade investigativa no Ensino de Ciências. Embora os licenciandos possam ter problemas iniciais com as atividades, após a conclusão é observável uma sensação de satisfação com êxito nas atividades, seguida de críticas a respeito dos projetos.

RIAEE - Revista Ibero-Americana de Estudos em Educação, Araraquara, v. 16, n. 4, p. 2872-2887, out./dez. 2021. e-ISSN: 1982-5587 
No campo do ensino e aprendizagem baseados em investigação, alguns autores sugerem que o desenvolvimento profissional na fase de formação inicial forneça aos professores experiências de pesquisa. Isso acontece porque os estudantes que passam por estes desafios podem desenvolver melhores práticas de ensino que efetivamente orientem seus estudantes durante o processo de investigação (KAZEMPOUR, 2009). Em adição, Sadeh e Zion (2009) sugerem que os licenciandos que praticam atividade de investigação exibem um domínio maior no desempenho da dinâmica e dos processos da investigação.

De acordo com os resultados, foi possível observar que o envolvimento dos licenciandos em uma atividade de investigação no Ensino de Ciências concentrou-se mais nas incertezas da atividade científica do que em outros aspectos no processo de investigação. Segundo Adler; Zion e Rimerman-Shmueli (2019), focar a atenção na incerteza envolvida no processo de investigação é um passo importante para sua capacitação na implementação das atividades baseadas em investigação na sala de aula e orienta efetivamente seus estudantes nos processos de aprendizagem incertos e imprevisíveis.

Os estudantes de licenciatura que participaram das atividades e apresentaram a reflexão discutiram o processo contínuo de uma atividade investigativa. Eles salientaram que tal atividade não termina quando o projeto termina, pois o contexto de cada estudante traz questionamentos que ainda precisam ser explorados. Eles também mencionam a necessidade de uma continuidade da atividade: "Seria interessante dar continuidade a atividade para poder ver os resultados ao longo do tempo" (EST 8). Desta forma, é importante que os professores abordem os questionamentos como um processo contínuo durante todo o processo de investigação, enquanto tentam encontrar uma solução para o problema científico a ser trabalhado (ADLER; ZION; RIMERMAN-SHMUELI, 2019). Com base nesses resultados, propomos que, em cursos de licenciatura, o pensamento contínuo subjacente ao processo de investigação seja proposital e explicitamente estruturado nas disciplinas pedagógicas. As reflexões dos participantes sugeriram uma compreensão do processo de investigação científica e uma maior disposição e senso de preparação para incorporar experiências de investigação no Ensino de Ciências em suas futuras salas de aula.

Considerando as reflexões desenvolvidas pelos licenciandos, a próxima categoria relaciona "as associações das disciplinas práticas com o exercício de uma atividade de investigação". Segundo Freitas e Villani (2016), os licenciandos são aprendizes que estão ativamente construindo visões sobre o ensino e aprendizagem baseados nas experiências pessoais desenvolvidas durantes experiências vividas. Desta forma, as experiências vividas 
nas escolas, por meio da pesquisa, permitem uma maior reflexão sobre as práticas pedagógicas, como retratado nos relatos a seguir:

Ter contato com a atividade proposta na disciplina contribuiu para uma melhor formação profissional. Mesmo que não sigamos nessa área, essa experiência muda nosso olhar dentro da sala de aula, pois aprendemos a observar e tentar entender o que estamos observando... nos tornando melhores professores (EST 25)

A experiência de pesquisa é uma forma ativa de estar produzindo conhecimento, e não apenas reproduzindo-o. Isso é muito valioso, e eu sinto que deveria ser mais incentivado dentro das instituições de ensino, pois nos retira da posição passiva no âmbito da educação e aprendizado (EST 36).

Alguns autores (DRESNER; WORLEY, 2006; MORRISON, 2008; ZION et al., 2018) sugerem que professores em formação podem desenvolver uma maior compreensão da ciência de investigação por meio de realizações individuais em seus cursos de licenciaturas. Portanto, os cursos que proporcionam tais experiências práticas de investigação podem fornecer ferramentas poderosas para orientar a capacidade dos futuros professores em orientar seus estudantes em um trabalho de investigação (ZION et al., 2018).

Neste cenário, Menezes (2017) afirma que a experiência da prática de pesquisa, assistida por reflexões sobre a docência, pode oferecer uma possibilidade para que o licenciando reflita sobre sua formação profissional mesmo diante das dificuldades. Vale ressaltar que, "nem todo professor, por ser reflexivo, é também pesquisador, embora a recíproca seja, por força, verdadeira" (LÜDKE, 2001, p. 31). As atividades de pesquisa sugerem que o pesquisador exerça uma postura reflexiva, desenvolvendo um senso crítico (LÜDKE, 2001).

A percepção que os discentes possuem sobre suas atividades evidencia a experiência vivenciada, corroborando com as considerações feitas por outros trabalhos (TARDIF; LESSARD; LAHAYE1991; THERRIEN, 1997). Tardif, Lessard and Lahaye (1991) destacam que os docentes conferem um estatuto individual aos saberes da experiência, no qual, por meio desses saberes, elaboram parâmetros para julgar o que consideram importante como competência profissional para docência.

Sobre as associações do exercício da pesquisa com as disciplinas de práticas alguns estudantes relataram:

A prática de uma atividade de investigação me despertou ainda mais o interesse pela educação... (EST 2).

A disciplina me permitiu ter um contato próximo com a investigação, onde tive que trabalhar em todas as etapas envolvidas no processo de pesquisa

RIAEE - Revista Ibero-Americana de Estudos em Educação, Araraquara, v. 16, n. 4, p. 2872-2887, out./dez. 2021. e-ISSN: 1982-5587 
envolvidos. Pude aprender muito também no contato com as discussões produzidas pelos meus estudantes. (EST 16).

Com um olhar mais critico sobre as atividades, posso entender que a educação é muito complexa. O empoderamento do conhecimento é a junção da teoria, prática, campo entre outras, com objetivos fixos e anteriormente pesquisados e analisados para enfim ocorrer o real aprendizado (EST 19).

A prática de pesquisa desenvolvida como uma das disciplinas pedagógicas do curso de Licenciatura em Ciências Biológicas possibilita ao estudante um aprimoramento profissional com inserção da pesquisa no Ensino de Biologia. Pensando como contribuir para a ampliação dos espaços de pesquisa e diminuir o espaço entre a instituição e a escola, considero a afirmação de Orso (2000):

Há um distanciamento entre o que se ensina e a realidade vivenciada pelos alunos. Esta Constatação leva a repensar o trabalho realizado na escola e na universidade, de tal forma que se contribua para superar essa fragmentação. Certo que não consigo compreender todos meus questionamentos, finalizo o trabalho com a convicção de que há muito a ser estudado no ensino de biologia (ORSO, 2000, p. 3).

Por fim, todos os estudantes descritos aqui também enfatizam as experiências práticas vivenciadas por meio da pesquisa no ensino de biologia como um requisito para a construção do conhecimento e aprendizado nas ciências. Essas observações contribuem para fomentar a prática da pesquisa em sala de aula, como também em outras práticas baseadas em questionamentos. Mais especificamente, sugerem a importância de oferta aos discentes que estão no processo de formação à docência oportunidades de vivenciar de forma prática essa estratégia didática, a fim de construir uma identidade profissional. Essa tendência dos licenciandos também foi encontrada na literatura (WINDSCHITL, 2003, MENEZES, 2017).

\section{Considerações finais}

Tentando compreender a contribuição das experiências de investigação na mudança de concepções dos estudantes de licenciatura foi observado que as disciplinas práticas proporcionam aos estudantes vivencias e experiências que modificam as concepções que os mesmos possuem sobre a investigação em sala de aula. Essas modificações são apresentadas na forma que os discentes refletem sobre o processo de pesquisa no Ensino de Ciências em sala de aula, aproximando-os da produção do conhecimento. Desta forma, é possível inferir que os licenciandos foram capazes de compreender os diferentes aspectos do aprendizado baseado em investigação. A nossa terceira pergunta relaciona a importância das disciplinas de Práticas de Ensino e como estas contribuem com os estudantes de licenciatura a adquirir 
habilidades no Ensino de Ciências baseado em investigação. Apesar de observar a importância que as disciplinas práticas possuem no desenvolvimento dos discentes, vale ressaltar que a pesquisa tem algumas limitações. A respeito dessas limitações menciona-se que os participantes do trabalho foram observados em situação de formação inicial e não em suas próprias salas de aulas, o tipo de interpretações dos resultados e o pequeno tamanho da amostra. Devido a essas limitações, não podemos determinar em que grau o uso da investigação em sala de aula desenvolveu uma melhor compreensão dos licenciandos sobre as principais características da investigação. Entretanto, nossos resultados demonstram que o envolvimento dos estudantes de licenciatura neste processo sustentou reflexões sobre as características dinâmicas da investigação.

\section{REFERÊNCIAS}

ADLER, I. et al. Reading between the lines: The effect of contextual factors on student motivation throughout an open inquiry process. Science Education, v. 102, n. 4, p. 820-855, 2018.

ADLER, I.; ZION, M.; RIMERMAN-SHMUELI, E. Fostering Teachers' Reflections on the Dynamic Characteristics of Open Inquiry through Metacognitive Prompts. Journal of Science Teacher Education, v. 30, n. 7, p. 763-787, 2019.

BARDIN, L. Content analysis. São Paulo: Edições, 2011. v. 70, p. 279.

BAXTER, B. K. et al. Using a multilevel assessment scheme in reforming science methods courses. Journal of Science Teacher Education, v. 15, n. 3, p. 211-232, 2004.

BHATTACHARYYA, S.; VOLK, T.; LUMPE, A. The influence of an extensive inquirybased field experience on pre-service elementary student teachers' science teaching beliefs. Journal of Science Teacher Education, v. 20, n. 3, p. 199-218, 2009.

BLANCHARD, M. et al. Is inquiry possible in light of accountability? A quantitative comparison of the relative effectiveness of guided inquiry and verification laboratory instruction. Science Education, v. 94, n. 4, p. 577-616, 2010.

BREDA, A. et al. A investigação como princípio educativo na formação de professores de Ciências e Matemática. Revista Caderno Pedagógico, v. 13, n. 1, 2016.

CAPPS, D. K.; CRAWFORD, B. A.; CONSTAS, M. A. A review of empirical literature on inquiry professional development: Alignment with best practices and a critique of the findings. Journal of science teacher education, v. 23, n. 3, p. 291-318, 2012.

CHEN, H.-T. et al. Longitudinal study of an after-school, inquiry-based science intervention on low-achieving children's affective perceptions of learning science. International Journal of Science Education, v. 36, n. 13, p. 2133-2156, 2014. 
CRAWFORD, B. A. Embracing the essence of inquiry: New roles for science teachers. Journal of Research in Science Teaching: The Official Journal of the National Association for Research in Science Teaching, v. 37, n. 9, p. 916-937, 2000.

CUNHA, R. S. et al. Formação inicial docente e suas relações dentro do âmbito escolar. Ciência \& Educação, v. 22, n. 3, p. 585-596, 2016.

DARLING-HAMMOND, L.; MCLAUGHLIN, M. W. Policies that support professional development in an era of reform. Phi delta kappan, v. 76, n. 8, p. 597-604, 1995.

DATTEIN, R. W.; DA COSTA GÜLLICH, R. I.; ZANON, L. B. Escritas reflexivas compartilhadas como estratégia de formação inicial: a pesquisa no ensino e na iniciação à docência em ciências. Ensino \& Pesquisa, v. 16, n. 1, 2018.

DRESNER, M.; WORLEY, E. Teacher research experiences, partnerships with scientists, and teacher networks sustaining factors from professional development. Journal of Science Teacher Education, v. 17, n. 1, p. 1-14, 2006.

FREITAS, D.; VILLANI, A. Formação de professores de ciências: um desafio sem limites. Investigações em Ensino de Ciências, v. 7, n. 3, p. 215-230, 2016.

GROSSMAN, P. L.; WILSON, S. M.; SHULMAN, L. S. Teachers of substance: Subject matter knowledge for teaching. Profesorado, Revista de currículum y formación del profesorado, v. 9, n. 2, p. 1-25, 1989.

HARRES, J. B. S. et al. Laboratórios de Ensino: inovação curricular na formação de professores de ciências. ESETec, v. 1, p. 99, 2005.

HMELO-SILVER, C. E.; DUNCAN, R. G.; CHINN, C. A. Scaffolding and achievement in problem-based and inquiry learning: a response to Kirschner, Sweller. Educational psychologist, v. 42, n. 2, p. 99-107, 2007.

HUBERMAN, A. M.; MILES, M. B. Data management and analysis methods. 1994.

KABERMAN, Z.; DORI, Y. J. Question posing, inquiry, and modeling skills of chemistry students in the case-based computerized laboratory environment. International Journal of Science and Mathematics Education, v. 7, n. 3, p. 597-625, 2009.

KAZEMPOUR, M. Impact of inquiry-based professional development on core conceptions and teaching practices: A case study. Science Educator, v. 18, n. 2, p. 56-68, 2009.

KAZEMPOUR, M. The Interrelationship of Science Experiences, Beliefs, Attitudes, and SelfEfficacy: A Case Study of a Pre-Service Teacher with Positive Science Attitude and High Science Teaching Self-Efficacy. European Journal of Science and Mathematics Education, v. 1, n. 3, p. 106-124, 2013.

LEE, O. et al. Urban elementary school teachers' knowledge and practices in teaching science to English language learners. Science Education, v. 92, n. 4, p. 733-758, 2008. 
LOUGHRAN, J. Bridging the gap: An analysis of the needs of second-year science teachers. Science Education, v. 78, n. 4, p. 365-386, 1994.

LÜDKE, M. A complexa relação entre o professor e a pesquisa. O papel da pesquisa na formação e na prática dos professores. Campinas: Papirus, p. 27-54, 2001.

MARSHALL, J. C. et al. Inquiry-based instruction: A possible solution to improving student learning of both science concepts and scientific practices. International journal of science and mathematics education, v. 15, n. 5, p. 777-796, 2017.

MCLAUGHLIN, C. A.; MACFADDEN, B. J. At the elbows of scientists: Shaping science teachers' conceptions and enactment of inquiry-based instruction. Research in Science Education, v. 44, n. 6, p. 927-947, 2014.

MENEZES, J. P. C. Importância de trabalhar práticas de ensino na formação inicial docente. In: Ciências da Natureza e Formação de Professores: entre desafios e perspectivas apresentados no CECIFOP 2017. 1. ed. 2017.

MORRISON, J. A. Individual inquiry investigations in an elementary science methods course. Journal of Science Teacher Education, v. 19, n. 2, p. 117-134, 2008.

MUNFORD, D.; LIMA, M. E. C. C. Ensinar ciências por investigação: em quê estamos de acordo? Ensaio Pesquisa em Educação em Ciências, Belo Horizonte, v. 9, n. 1, p. 89-111, 2007.

\section{NATIONAL RESEARCH COUNCIL. Inquiry and the national science education} standards: A guide for teaching and learning. National Academies Press, 2000.

OLIVEIRA, J. M. P.; SCHNEIDER, E. M. Os projetos de trabalho: uma alternativa na formação inicial para a articulação teórico-prática. Revista de educación en biología, v. 19, n. 1, p. 19-34, 2016.

ORSO, P. J. Educação e saúde: a interdisciplinaridade como desafio. ETD-Educação Temática Digital, v. 2, n. 1, p. 1-5, 2000.

PREDEBON, F.; DEL PINO, J. C. Uma análise evolutiva de modelos didáticos associados às concepções didáticas de futuros professores de química envolvidos em um processo de intervenção formativa. Investigações em Ensino de Ciências, v. 14, n. 2, p. 237-254, 2016.

ROGERS, M. A. P. Elementary preservice teachers' experience with inquiry: Connecting evidence to explanation. Journal of Elementary Science Education, v. 21, n. 3, p. 47, 2009.

ROMERO-ARIZA, M. et al. Highly Recommended and Poorly Used: English and Spanish Science Teachers' Views of Inquiry-Based Learning (IBL) and Its Enactment. EURASIA Journal of Mathematics, Science and Technology Education, v. 16, n. 1, 2020.

SAAD, R.; BOUJAOUDE, S. The relationship between teachers' knowledge and beliefs about science and inquiry and their classroom practices. Eurasia Journal of Mathematics, Science \& Technology Education, v. 8, n. 2, p. 113-128, 2012. 
SADEH, I.; ZION, M. The development of dynamic inquiry performances within an open inquiry setting: A comparison to guided inquiry setting. Journal of Research in Science Teaching: The Official Journal of the National Association for Research in Science Teaching, v. 46, n. 10, p. 1137-1160, 2009.

SASSERON, L. H. Ensino de ciências por investigação e o desenvolvimento de práticas: uma mirada para a base nacional comum curricular. Revista Brasileira de Pesquisa em Educação em Ciências, p. 1061-1085, 2018.

SHULMAN, L. S. Those who understand: Knowledge growth in teaching. Journal of Education, v. 193, n. 3, p. 1-11, 2013.

STAFFORD, J. R. O currículo e o trabalho por meio de projetos: construindo alternativas pela prática investigativa. Regae-Revista de Gestão e Avaliação Educacional, v. 5, n. 10, p. 55 64, 2016.

TARDIF, M.; LESSARD, C.; LAHAYE, L. Esboço de uma problemática do saber docente. Teoria \& Educação, v. 1, n. 4, p. 215-253, 1991.

THERRIEN, J. Saber de experiência, identidade e competência profissional: como os docentes produzem sua profissão. Contexto \& Educação, v. 12, n. 48, p. 7-36, 1997.

VÁZQUEZ-ALONSO, Á.; MANASSERO-MAS, M.-A. Hacia una formación inicial del profesorado de ciencias basada en la investigación. Revista Española de Pedagogía, p. 343$363,2015$.

WINDSCHITL, M. Inquiry projects in science teacher education: What can investigative experiences reveal about teacher thinking and eventual classroom practice?. Science Education, v. 87, n. 1, p. 112-143, 2003.

ZION, M. et al. Supporting Teachers' Understanding of Nature of Science and Inquiry Through Personal Experience and Perception of Inquiry as a Dynamic Process. Research in Science Education, p. 1-24, 2018.

\section{Como referenciar este artigo}

MENEZES, J. P. C. Concepção sobre a investigação no ensino de ciências. Revista IberoAmericana de Estudos em Educação, Araraquara, v. 16, n. 4, p. 2872-2887, out./dez. 2021. e-ISSN: 1982-5587. DOI: https://doi.org/10.21723/riaee.v16i4.13688

Submetido em: 01/07/2021

Revisões requeridas em: 04/08/2021

Aprovado em: 11/09/2021

Publicado em: 21/10/2021 